\title{
Optimization of Flexible Assembly Systems for Electrical Motors
}

\author{
Mirlind Bruqi ${ }^{1}$, Ramë Likaj ${ }^{1}$, and Jorgaq Kaqani ${ }^{2}$ \\ ${ }^{1}$ Faculty of Mechanical Engineering, Kosovo \\ \{mirlind.bruci, rame.likaj\}@uni-pr.edu \\ ${ }^{2}$ Polytechnic University of Tirana, Albania
}

\begin{abstract}
The modern assembly systems are fully integrated production systems. They consist of interconnected components of hardware and software. Large flexible assembly systems are being composed of high number of components and managing requires an enormous volume of data. For that reason the controlling and optimization of such systems is too complex. Nowadays that can achieved, using just computers and software tools. In this paper the optimization of product mix for assembly system for electric motors will be described. Also special attention will be given to data security of the information system using cryptography.
\end{abstract}

Keywords: Flexible assembly, optimization, simulation, analysis software, cryptography.

\section{$1 \quad$ Introduction}

Simulation as a modern concept for system analysis, in particular for production systems is driven by rapid technological development of IT. Various experts have developed numerous algorithms, implementing them in various programming languages. Nowadays an engineer's task is to teach the simulation philosophy in order to be set free from very complex mathematical expressions, creating more space for individual creativity and engineering.

However for simulation of processes, other knowledge is required depending on the field or the level of research.

If we are in a level of a Decision Maker, which requires decisions to be made concerning manufacturing decisions etc, the decision maker should have knowledge of statistics, theory of probability, and a number of other theories linked to buffering and services. Fortunately, modern simulation programs are very advanced, and integrated program packages for statistical preparation of production data and automatic optimization of entire process are included in them. In many cases such simulation process for production system allows very fast and efficient analysis of the stability for the selection of its parameters.

Arena is a simulator, which enables fast and efficient modelling of wide natural and artificial systems. It is a language which is designed for analysis and simulation of a large number of production and logistic systems, warehouses and all service systems. Service systems include: restaurants, post offices, banks, hospitals etc. 


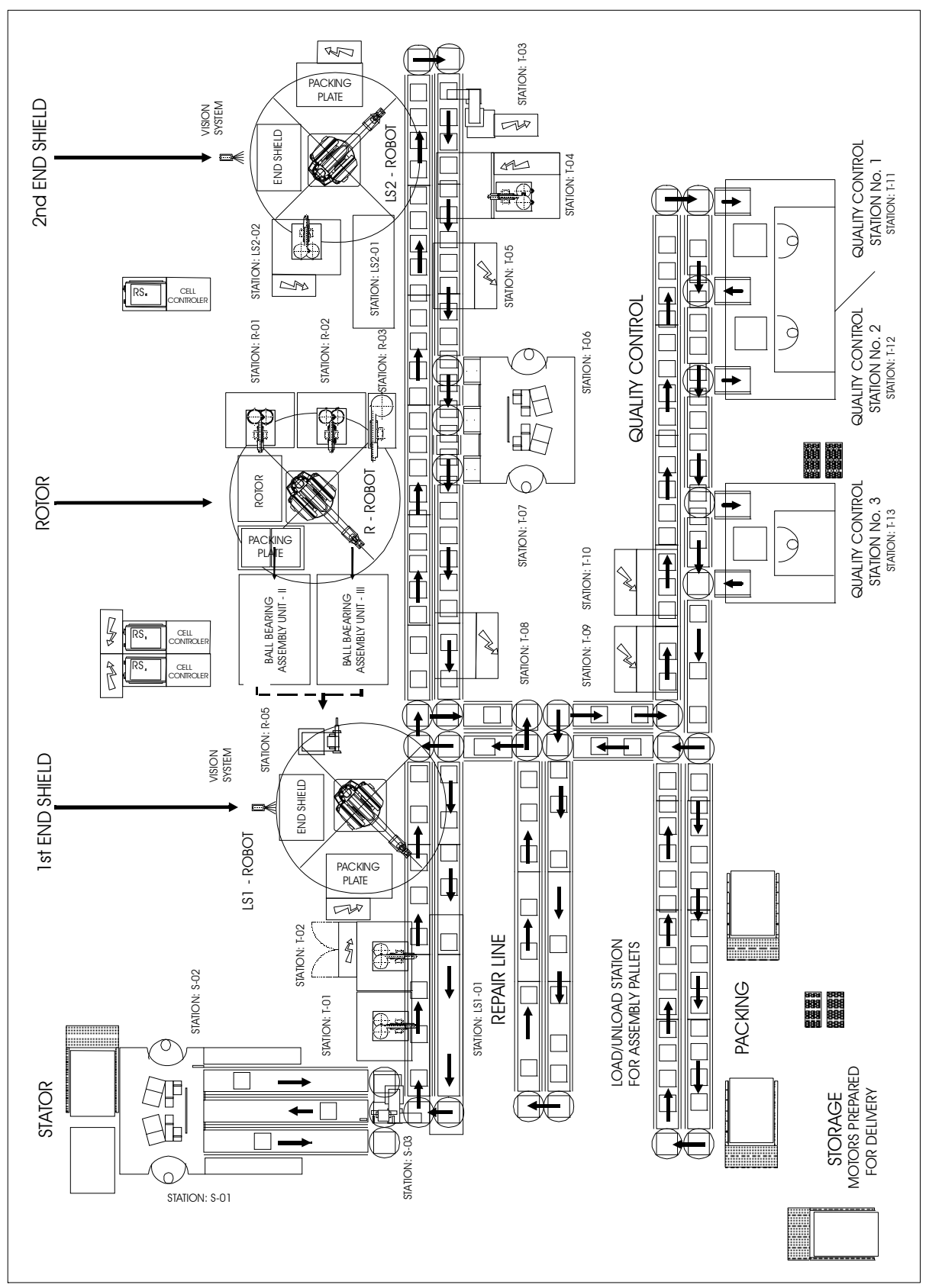

Fig. 1. Flexible system of electro-motor's assembling 
Systems which can be analysed by Arena are listed below:

- Detailed analysis of each type of production systems, transportation devices and industrial robots,

- Analysis of complex service systems and customer relationship management systems,

- Global analysis of a Supply Chain which include, warehousing, transportation and logistic,

- Prediction of system performance based on key parameters such as; cost, quantity, cycle timing, and system efficiency,

- Identification of bottlenecks of the Queuing systems and over exploitation of system resources,

- $\quad$ Resource planning of staff, equipments and facilities, materials etc.

By using Arena and its template the following can be achieved:

- Modelling of relevant processes within imaginary system boundaries,

- Simulation of system in order to understand and interpret complex relationship between its elements, identification of strategic points of production segments,

- Visualisation of system operations by graphic animation in order to facilitate both simulation steps, verification and validation,

- Analysis of the current state of the system "as it is" or "how it might be", in order to make better planning for the future.

\section{System Analysis and Goal Defining, the Aim of Simulation}

Activities at the flexible system of the electrical machines assembling are very complex, and need to be described in detail. For the purposes of modelling, only main points of them will be subject of analysis.

The system which is considered for analysis from the entry at the first conveyor as a resource point up to the exit from conveyor as an absorbing point for the parts, and is built by following resources:

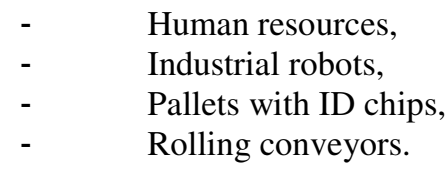

In Figure 1 is shown graphically the path of all entities that circulate in the flexible assembling system. The path of material flux through working stations contains following elements:

- $\quad$ Input / Output points of the system given by rectangle,

- $\quad$ Stations given by small circle denoted by R (Resource),

- Logic points or system nodes given as parallelepiped (N-Node),

- $\quad$ Straight lines represent the conveyors,

- Arrows show the direction of the material flux. 


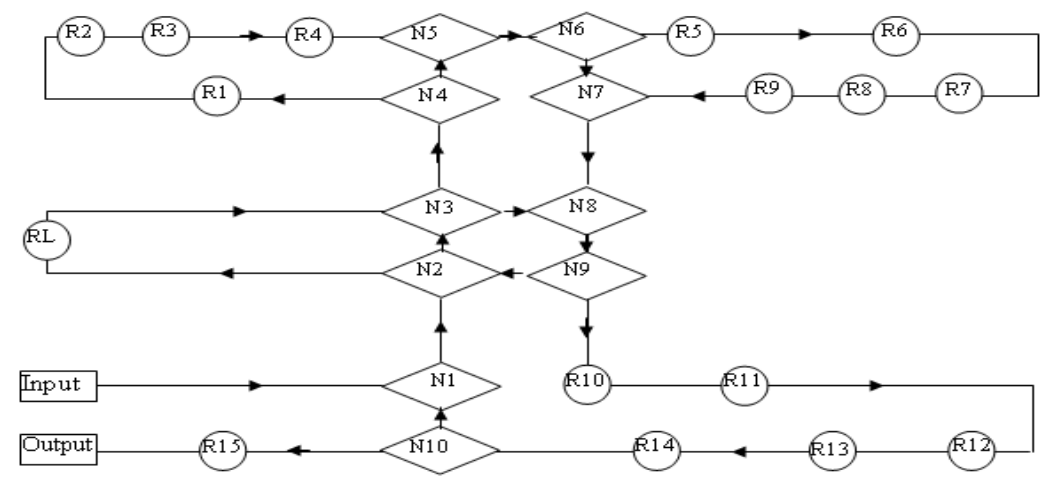

Fig. 2. Scheme of material flow through the assembling flexible system

System input represents the source of entities, indicating the input boundary of the system, while output or so-called disappearance point of entities is output boundary of the system. But, the subject of study is analysis of the inner part of the system by considering that all external activities are reduced in two points, input and output. Such approximation brings to the definition the system boundaries.

System allows change the number of resources at working station and also change of material flux depending on overfeed of the system with pallets and to change the frequency of material flux. Therefore, the system input and number of the engaged resources are considered as variables. In the other side, the system is influenced by different demands of the consumers which are made in different timing and in various quantities. These are representing stochastic effects that interfere and put condition to the system itself. The aim of the simulation is to achieve a maximum level of rent ability based on these demands, conditions, schedule and resources.

It is adopted that:

$C_{R i}$ - are the costs for $i$ resources for the simulation period, $i=1, \ldots, n$,

$C_{M i^{-}}$are the material costs of specified product $i$, for $i=1$ to $n$ (Euro)

$C_{T i}$ - are the costs for waiting time of product-Entity Flow Time $i$ of the system, for $i=1$ to $n$ (Euro)

$\Psi$ - is the objective function.

By the simulation process we are trying to minimise the objective function. This means that if decrease of the costs is achieved the main goal of optimisation is met, meaning that "with minimum utilisation of resources the maximum production volume is obtained, satisfying technical and organization conditions of the system".

Mathematical model is expressed by:

$$
\min (\Psi)=\min \left[\sum_{i=1}^{n} \mathrm{C}_{\mathrm{Ri}}+\sum_{i=1}^{n} C_{\mathrm{Mi}}+\sum_{i=1}^{n} C_{\mathrm{Ti}}\right]
$$

If $k$ - simulations are executed then function (1) has form as given in (2): 


$$
\min \left(\sum_{j=1}^{k} \Psi_{\mathrm{j}}\right)=\min \left\{\sum_{j=1}^{k}\left[\sum_{i=1}^{n} \mathrm{C}_{\mathrm{Rij}}+\sum_{i=1}^{n} C_{\mathrm{Mij}}+\sum_{i=1}^{n} C_{\mathrm{Tij}}\right]\right\}
$$

So, the simulation that in the best way fulfils the criteria is adopted. This operation is realized with OptQuest package which will be described later.

At OptQuest is chosen the option:

$$
\min \left(\sum_{j=1}^{k} \Psi_{\mathrm{j}}\right) \approx=\operatorname{minimize}\{\text { Entity.Flow.Time }\}
$$

In this case, the only goal was the definition of the objective for our experiments in the assembling flexible system.

A flexible assembly system is an extremely complex production system. This attribute of the system can be described as the reaction of the market, which forces the manufacturer for decreasing a time to bring up the variety products to market. To accomplish this task, the flexible assembly system must quickly respond to the needs of the market. In this case, the system must be able to reconfigure his structure very quickly. These requirements have a large influence on the organization and technology of a production. Effect of these demands is constantly growing complexity and forces the manufactures to strengthen the flexibility of manufacturing and assembling systems.

To achieve these goals, systems must accomplish the following tasks:

- Increase productivity.

- Increase the quality of products and handle integrated quality management with higher efficiency.

- Higher utilization of machines, and stations.

- Quick respond on the external influences and demands.

In order to achieve these tasks and to reduce the complexity of the system itself, the complex global objectives can be transformed into a many simple solutions, which are able to meet local objectives.

These local objectives are based on hierarchical decomposition of time horizon. In this hierarchical framework there are some interconnected decision levels:

- $\quad$ Flexible assembly system for design (long term).

- Flexible assembly system for planning (medium term).

- Flexible assembly system for scheduling and control (short term).

The long term of time horizon means, the period of time on which the decision for design of system is long enough. In that case, in order to reduce the volume of data and the complexity of design process, a lot of activities will be integrated in single assumed activity.

The short term of time horizon means, the period of time by which the decision about system and its components will be made. This short term of time horizon give us an answer about the allocation of system resources in a very short period of time. 
In this paper system is presented the simulation model for electrical motors assembly. This model gives the answer about system behavior. Simulation is made in simulation package ARENA. With OptQuest for Arena is carried out the optimization of product mix.

\section{Description of Flexible Assembly System}

The core system consists of industrial robots, assembly stations, transport systems and assembly palettes with ID and memory component. The Assembly stations are thought to be automatic transport systems with few integrated assembly sub-systems. Assembly systems are closed cycle. The assembly pallet is the basic carrier for a product during the assembly cycle. The assembly pallets are moved by conveyer belt system. After each assembly step the quality control check is done. If the state of quality check is positive, then the next assembly operation takes place. If the state of quality check is negative, then palette has to go to the repair station and wait for the shop floor operator. Shop floor operator must try to repair the product. If this is not possible he/she has to remove its content from the system, reset the empty pallet and send it in the direction of loading/unloading station. If repair is done, operator must proceed with palette ID and send it to the assembly cycle. New ID corresponds to the current state of product. Palette with repaired product will be rejected and move to the correct assembly station which is defined by given ID.

After completing the assembly cycle, palette must be in a loading/unloading station. After that, finished product will be unloaded and palette will be given a new status. This system is shown in fig. 3 .

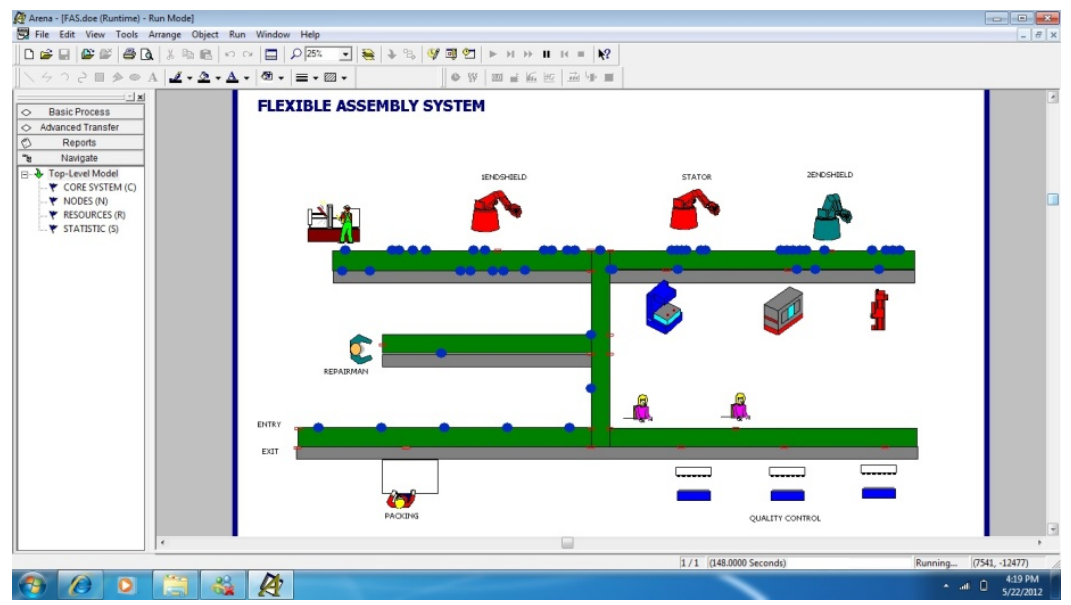

Fig. 3. Flexible assembly system 


\section{$4 \quad$ Entering Samples for Flexible System for Assembling}

Another important point of the simulation is to generate data for the working stations known as samples, which is done in a manual and automatic way. Once the system is equipped by a device for identification of pallets and because the system has the possibility of communication with central computer, in this way there is possibility for automatic tracking of data. The data that must be entered in this case are listed in the following:

- Time of arrival of piece at the x station and type,

- Time of piece processing at the station,

- The time between the fall of assembling system and the duration of the fall,

- Schedule of the system, short and long breaks, changes and other organisational restrictions.

\section{$5 \quad$ Basic Characteristics of Flexible Assembly Systems}

The Simulated flexible complex assembly system deals with following sources of uncertainty and dynamic events:

- Variable availability, arrival rates and has the incoming parts.

- Variable quality of incoming parts - defective parts have to be removed before, either causing errors in system, or being assembled into finished products.

- $\quad$ Mixed batch of parts to be assembled - The assembly station assembling a range of variants of electrical motors types.

- Variable availability of resources - resources are tools and machines by which the assembly station must be coordinated directly.

- Response to dynamic events in assembly system - For example opening alternative assembly stations to bypass machine breakdowns or allowing more palettes to increase production capacity.

\section{Optimisation Using Optquest for Arena}

OptQuest for Arena is designed for manufacturing or business process consultants, analysts and industrial or systems engineers. It is typically deployed as an enterprise business analysis and productivity tool. OptQuest enhances the analysis capabilities of Arena allowing searching for optimal solutions within simulation models. In order to optimize the flexible assembly system in a case study, first the parameters have to be selected which have the largest influence on the product costs. In this case the system is very sensitive to these two parameters:

1. The number of operators in stations, and

2. The number of products on the batch mixes.

Constrains and profits for each Motor type are resented in Table 1: 
Table 1. Constrains and profits for each Motor type

\begin{tabular}{|l|l|l|l|l|l|}
\hline \multicolumn{5}{|l}{ Product Type } \\
\hline & A & B & C & D & E \\
\hline $\begin{array}{l}\text { Constraints } \\
{[\% \text { in mix }]}\end{array}$ & $\geq 13$ & $\geq 10$ & $\geq 12$ & $\geq 25$ & $\geq 30$ \\
\hline $\begin{array}{l}\text { Costs } \\
{[\text { Euro / Part }]}\end{array}$ & 17 & 13 & 15 & 28 & 32 \\
\hline
\end{tabular}

The numbers of resources in different stations may vary, as shown in Table 2.

Table 2. Variable number of resources per station

\begin{tabular}{|l|l|l|l|l|l|}
\hline Stations & S6 & S10 & S11 & S12 & Repair Line \\
\hline $\begin{array}{l}\text { Number of } \\
\text { Resources }\end{array}$ & 2 to 3 & 1 to 2 & 2 to 3 & 1 to 2 & 3 to 4 \\
\hline
\end{tabular}

These constraints can be added very easily to OptQuest for Arena and the optimization process can begin.

\section{$7 \quad$ Optimisation Results}

This paper presents simulations model of existing complex FAS.

Simulation model consists of 15 different stations. Additionally, quality control of the system is performed after each assembly station. Purpose of the simulation model is to find the best product mix and optimal numbers of replications on OptQuest for Arena, results are acquired and presented in the following tables (table 3 and 4).

Table 3. The optimal number of resources on stations

\begin{tabular}{|l|c|c|c|c|c|}
\hline Stations & S6 & S10 & S11 & S12 & Repair Line \\
\hline Number of Resources & 2 & 2 & 3 & 1 & 3 \\
\hline
\end{tabular}

Table 4. The optimal number of products on batch mixes

\begin{tabular}{|l|l|l|l|l|}
\hline \multicolumn{5}{|l|}{ Product Type on batch mix } \\
\hline A & B & C & D & E \\
\hline 15 & 10 & 12 & 33 & 30 \\
\hline
\end{tabular}

System security is achieved by using cryptographic methods, by using an algorithm which is designed specifically for FAS. Figure 4 represents an interface for system connection. 


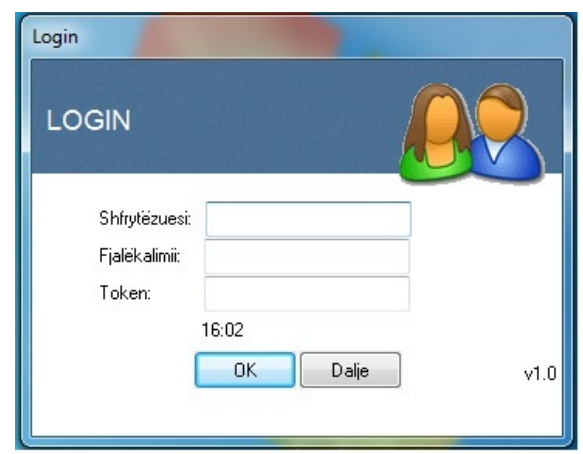

Fig. 4. Interface FAS connection

The application enables the encryption of various types of files such as "Doc, pdf, doe, dwg etc".

The application features are listed in the following:

- Symmetric encryption 256bit

- Usage of a non-public hash key

- Fast encryption/decryption of large files

- Intel x86 platform based on Windows Operating System

- Non-public algorithm

\section{Conclusions}

Flexible systems for assembling are part of the most sophisticated systems of the time. As such they have the possibility to pass from one assembly program to another in a very short period of time. The flexibility of such systems is its main property. In this way the Passover from one assembly system to another is made in: automatic, semi-automatic way or manually, by replacing the auxiliary devices of robots and machines.

Besides the positive side, these systems also have their negative side. Very often different products are mounted to these systems, and from managerial point of view this is heavy workload because of prior planning and preparations. These preparation tasks include: choosing an optimal or suboptimal scenario of material, energy and information flow, including human resource organization.

The selection of such scenarios is made through different optimization algorithms such as: finite, limited and random enumeration. Module varies from one system to another, thus this module must be built for each specific system.

The aim of this Paper is to introduce the OptQuest toolbox as new feature of Arena environment for the optimization of complex production systems. The algorithms for the optimization are described in Rockwell Automation handbook OptQuest for Arena.

This paper describes on of the easiest ways for optimization of flexible assembly systems using the OptQuest for Arena. The Results of this study are presented in tables 3 and 4. 


\section{References}

1. Bruçi, M., Stopper, M., Bunjaku, A., Stuja, K., Kubat, A.: Optimisation of Flexible Assembly System for Electrical Motors using Opt quest for Arena. In: DAAAM International, Vienna, Austria, pp. 071-072 (2003)

2. Bruçi, M., Bunjaku, A., Stuja, K., Buza, S.: Optimizing of Flexible Palletizing Lines Using Simulation Tools. In: TMT 2005, Antalya, Turkey, pp. 693-696 (2005)

3. Stuja, K., Stopper, M., Bunjaku, A., Bruçi, A.: A concept for scheduling of flexible assembly for electrical motors. In: DAAAM International Scientific Book, Vienna, Austria, pp. 591-598 (2003) ISBN 3-901509-36, ISSN 1726-9687

4. Katalinic, B.: Industrieroboter und flexible Fertigungssysteme für Dehteile. VDI-Verlag, Düsseldorf (1990) ISBN 318-401027-9

5. Rockwell Software, OptQuest for Arena- User's Guide.@ 2002 Rockwell Software. Inc., A Rockwell Automatic company, and Optimization Technologies (2002) 